\title{
Identification of dicarbonyl and L-xylulose reductase as a therapeutic target in human chronic kidney disease
}

Paul Perco, ${ }^{1}$ Wenjun Ju, ${ }^{2}$ Julia Kerschbaum, ${ }^{1}$ Johannes Leierer, ${ }^{1}$ Rajasree Menon, ${ }^{2}$

Catherine Zhu, ${ }^{2}$ Matthias Kretzler, ${ }^{2}$ Gert Mayer, ${ }^{1}$ Michael Rudnicki, ${ }^{1}$ and Nephrotic Syndrome Study Network (NEPTUNE)

'Department of Internal Medicine IV (Nephrology and Hypertension), Medical University Innsbruck, Innsbruck, Austria. 2Department of Internal Medicine, Department of Computational Medicine and Bioinformatics, University of Michigan, Ann Arbor, Michigan, USA. ${ }^{3}$ The Nephrotic Syndrome Study Network is detailed in the Supplemental Acknowledgments.

\begin{abstract}
An imbalance of nephroprotective factors and renal damaging molecules contributes to development and progression of chronic kidney disease (CKD). We investigated associations of renoprotective factor gene expression patterns with CKD severity and outcome. Gene expression profiles of 197 previously reported renoprotective factors were analyzed in a discovery cohort in renal biopsies of 63 CKD patients. Downregulation of dicarbonyl and L-xylulose reductase (DCXR) showed the strongest association with disease progression. This significant association was validated in an independent set of 225 patients with nephrotic syndrome from the multicenter NEPTUNE cohort. Reduced expression of DCXR was significantly associated with degree of histological damage as well as with lower estimated glomerular filtration rate and increased urinary protein levels. DCXR downregulation in CKD was confirmed in 3 publicly available transcriptomics data sets in the context of CKD. Expression of DCXR showed positive correlations to enzymes that are involved in dicarbonyl stress detoxification based on transcriptomics profiles. The sodium glucose cotransporter-2 (SGLT2) inhibitors canagliflozin and empagliflozin showed a beneficial effect on renal proximal tubular cells under diabetic stimuli-enhanced $D C X R$ gene expression. In summary, lower expression of the renoprotective factor DCXR in renal tissue is associated with more severe disease and worse outcome in human CKD.
\end{abstract}

Authorship note: PP and W] contributed equally to this work.

Conflict of interest: W] and MK are listed as inventors on a patent application (PCT/EP2014/073413) on biomarkers for CKD progression encompassing urinary EGF as a biomarker of CKD progression. MK's research group is supported by grants from the NIH, European Union Innovative Medicine Initiative, JDRF, AstraZeneca, Eli Lilly, Novo Nordisk, Coldfinch Bio, Elpidera, and Boehringer Ingelheim.

Copyright: () 2019, American Society for Clinical Investigation.

Submitted: February 22, 2019

Accepted: May 16, 2019

Published: June 20, 2019

Reference information: JCI Insight. 2019;4(12):e128120. https://doi. org/10.1172/jci.insight.128120.

\section{Introduction}

Chronic kidney disease (CKD) affects $7 \%-12 \%$ of the general population, and progression of $\mathrm{CKD}$ is associated with increased morbidity and mortality $(1,2)$. Established clinical risk factors for progression to end-stage renal disease (ESRD) are reduced eGFR, increased levels of proteinuria and albuminuria, as well as the presence of diabetes and hypertension. Histological hallmarks of progressive loss of renal function include infiltration of the kidney with inflammatory cells, activation and proliferation of fibroblasts, excessive production and deposition of extracellular matrix components, and rarefaction of tubular capillaries, finally resulting in irreversible tubulointerstitial fibrosis and atrophy $(3,4)$. At the molecular level, these processes are triggered by and lead to de novo intrarenal expression of miRNAs and mRNA transcripts representing damaging as well as protective/regenerative mechanisms (5). Traditionally, research has focused on the activation of deleterious factors and pathways that drive CKD progression.

We have previously consolidated a set of proteins with renoprotective potential based on scientific literature (6). A few of these factors are actually direct drug targets of therapies, such as the vitamin D receptor $(V D R)$, the glucagon-like peptide 1 receptor $(G L P 1 R)$, or the peroxisome proliferator-activated receptor $\gamma$ $(P P A R G)$. Other factors are currently investigated in clinical trials in the context of diabetes and kidney disease such as the hepatocyte growth factor (HGF) or proopiomelanocortin (POMC) encoding among others for the polypeptide hormone adrenocorticotropin. Proteins are, therefore, included in the set of renoprotective factors and hold the potential to serve as drug targets, biomarkers, or drugs themselves in the context of kidney disease. Identification of biomarkers most often focuses on molecules with damaging properties being elevated in the diseased state. A systematic evaluation of the association of disease progression with renoprotective factors has not been conducted so far to our knowledge. 
Table 1. Patient characteristics of the discovery cohort

\begin{tabular}{lcccc}
\hline & Cohort $(\boldsymbol{n}=\mathbf{6 3})$ & Progressive $(\boldsymbol{n}=\mathbf{2 4})$ & Stable $(\boldsymbol{n}=\mathbf{3 9})$ & $\boldsymbol{P}$ value \\
Age $(\mathrm{yr})$ & $47.2 \pm 17.5$ & $56.2 \pm 13.9$ & $41.7 \pm 17.3$ & 0.00057 \\
eGFR (ml/min/1.73 $\left.\mathrm{m}^{2}\right)$ & $63.3 \pm 35.2$ & $49.5 \pm 32.7$ & $71.7 \pm 34.4$ & 0.01335 \\
UPCR (g/g) & $4.4 \pm 3.4$ & $4.2 \pm 2.8$ & $4.5 \pm 3.7$ & 0.78860 \\
Follow-up time (yr) & $7.0 \pm 4.3$ & $4.7 \pm 3.2$ & $8.4 \pm 4.3$ & 0.00024 \\
Female sex [n (\%)] & $27(43)$ & $5(21)$ & $22(56)$ & 0.01418 \\
Baseline medication [n (\%)] & $29(46)$ & $13(54)$ & $16(41)$ & 0.33380 \\
RAAS blockade & $5(8)$ & $3(13)$ & $2(5)$ & $7(18)$ \\
Antidiabetics & $13(21)$ & $6(25)$ & $9(23)$ \\
Statins & $12(19)$ & $3(13)$ & $2(5)$ \\
Steroids & $2(3)$ & $0(0)$ & $4(10)$ \\
NSAIDs & $10(16)$ & $6(25)$ &
\end{tabular}

Key clinical parameters of the discovery cohort are given for the whole cohort as well as for the stable and progressive subgroups. The $t$ test was used for continuous and the $\chi^{2}$ test was used for discrete clinical parameters when comparing the stable and progressive patient groups. Mean values plus standard deviations are provided for continuous parameters. eGFR, estimated glomerular filtration rate; UPCR, urine protein to creatinine ratio; RAAS, renin angiotensin aldosterone system; NSAIDs, nonsteroidal antiinflammatory drugs.

In this study, we investigated a set of renoprotective factors on the transcriptome level and determined their association with disease outcome in a discovery cohort of $63 \mathrm{CKD}$ patients in the search for novel therapeutic targets. Findings were validated in independent CKD patient cohorts, including the multicenter and multiethnic Nephrotic Syndrome Study Network (NEPTUNE) cohort of 225 patients. The most promising candidate, $D C X R$, was further investigated on a functional level making use of human and in vitro transcriptomics data sets.

\section{Results}

In the current study, we investigated 187 proteins with renoprotective potential based on findings from the literature.

Clinical drivers of disease progression in the CKD discovery cohort. A retrospective cohort of 63 patients with various CKD diagnoses and available follow-up data was used as the discovery cohort to evaluate the association of renoprotective factors with CKD progression. Twenty-four of the sixty-three CKD patients developed end-stage renal disease (ESRD) - defined as the need for dialysis or renal transplantation - or experienced a 2-fold increase of serum creatinine and were therefore categorized as the progressive group. The median follow-up time was 6.9 years. At biopsy, patients in the progressive group, as compared with the group of stable patients, were older (56 years versus 41 years on average) and also had lower eGFR $\left(49.54 \mathrm{ml} / \mathrm{min} / 1.73 \mathrm{~m}^{2} \mathrm{vs} .71 .73 \mathrm{ml} / \mathrm{min} / 1.73 \mathrm{~m}^{2}\right)$. The percentage of male patients was also higher in the progressive group (Table 1).

Different diagnoses were represented in the set of 63 patients, with lupus nephritis (LN, $n=11)$, minimal change disease ( $\mathrm{MCD}, n=10)$, and membranous nephropathy $(\mathrm{MN}, n=10)$ being the 3 most frequent disease entities. Type of diagnosis was associated with outcome $(P<0.0001)$ in Kaplan-Meier analysis. Whereas urinary protein to creatinine ratio (UPCR) was not associated with disease progression, both sex $(P=0.0062)$ as well as eGFR at time of biopsy $(P=0.0033)$ were significantly associated with the course of disease, with male patients and patients with lower eGFR values having worse prognosis in Kaplan-Meier analysis. The list of patient IDs and group assignment is available in Supplemental Table 1.

Association of renoprotective factors with disease outcome in the CKD discovery cohort. Six renoprotective factors that were significantly downregulated in progressive patients were identified after statistical analysis of microarray (SAM) analysis: dicarbonyl and L-xylulose reductase ( $D C X R)$, epidermal growth factor $(E G F)$, glutathione S-transferase mu 1 (GSTM1), kininogen 1 (KNG1), nitric oxide synthase 3 (NOS3), and uromodulin (UMOD).

All 6 proteins were also significantly associated with outcomes in Kaplan-Meier analysis when grouping patients into tertiles based on marker expression levels (Figure 1). The lowest $P$ value after log-rank test was observed for $D C X R(P<0.0001)$. 

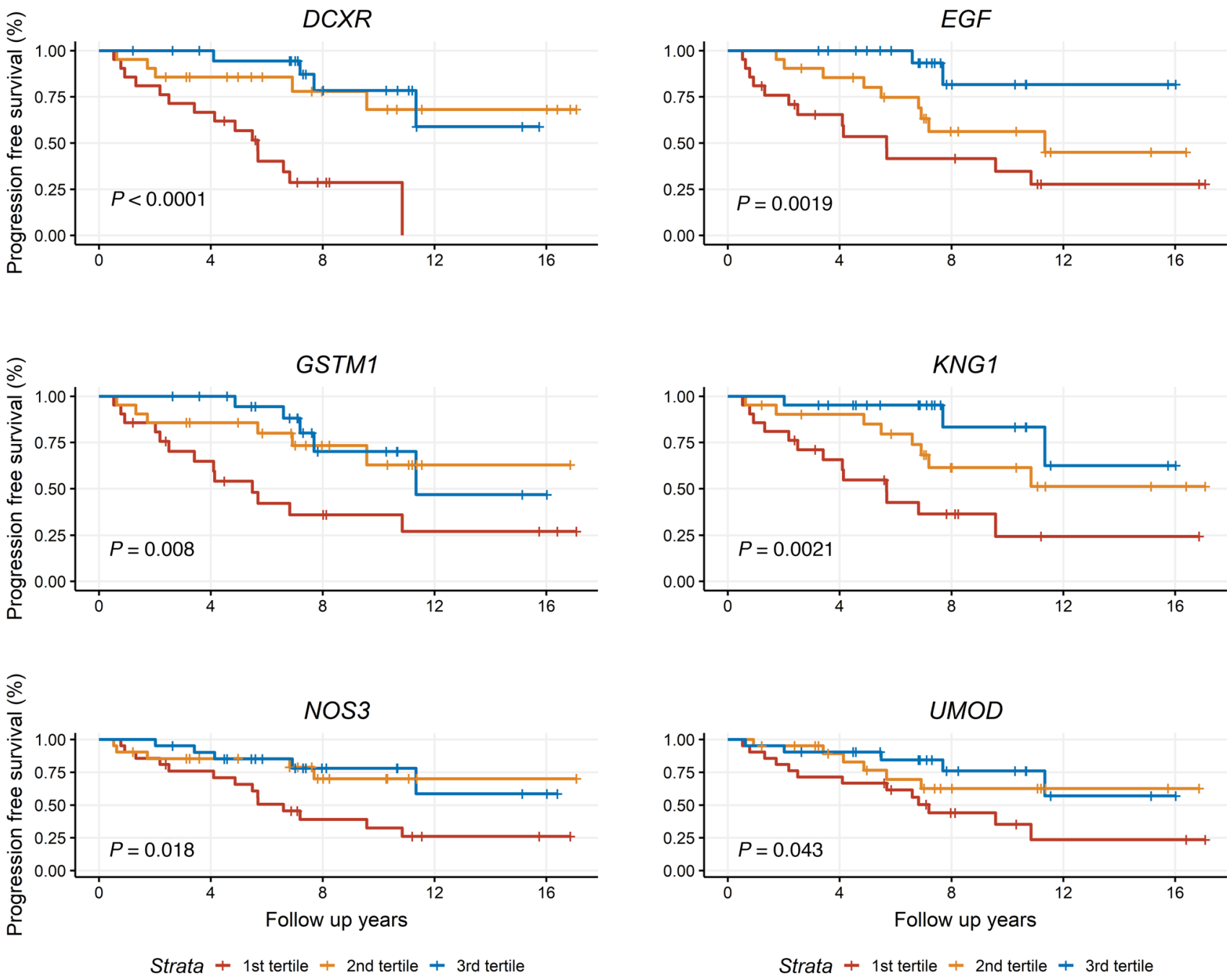

Figure 1. Association of renoprotective factors with disease outcome in the discovery cohort. Kaplan-Meier plots for the 6 renoprotective factors showing significant associations with disease outcome (log-rank test). Patients $(n=63)$ were divided into tertiles based on expression levels of the respective renoprotective factors.

EGF, KNG1, GSTM1, and NOS3 all showed significant positive correlations with baseline eGFR values (Figure 2). However, the correlation coefficient of $D C X R$ with baseline eGFR was 0.31 was not significant after correction for multiple testing. $D C X R$ showed significant positive correlations with $E G F$ $($ rho $=0.41)$ and $K N G 1($ rho $=0.59)$. UPCR did not show a marked correlation to any of the other parameters under study.

With available biomarker evidence in scientific literature for EGF, KNG1, GSTM1, NOS3, and UMOD, we focused in all further analyses on $D C X R$, for which no biomarker information in the context of human $\mathrm{CKD}$ was found in the literature.

$D C X R$ expression across different $C K D$ diagnoses and association with degree of histological damage. Expression levels of $D C X R$ were evaluated across the different disease diagnoses in the CKD discovery cohort. The lowest median $D C X R$ expression levels were found in the set of patients with membranoproliferative glomerulonephritis (MPGN), vasculitis, and diabetic nephropathy (DN). The highest median $D C X R$ expression values were found in the set of minimal change disease (MCD) patients, who all showed a stable course of disease (Figure 3A).

We observed an overall trend of lower $D C X R$ values being present in samples with a higher degree of histological damage. Significant associations were identified for interstitial inflammation $(P<0.001)$ and interstitial fibrosis $(P=0.0157$; Figure 3B).

$D C X R$ is predominantly expressed in the tubulointerstitium. In order to determine the levels of $D C X R$ transcript and protein abundance in the different renal compartments, we assessed $D C X R$ abundance in 3 


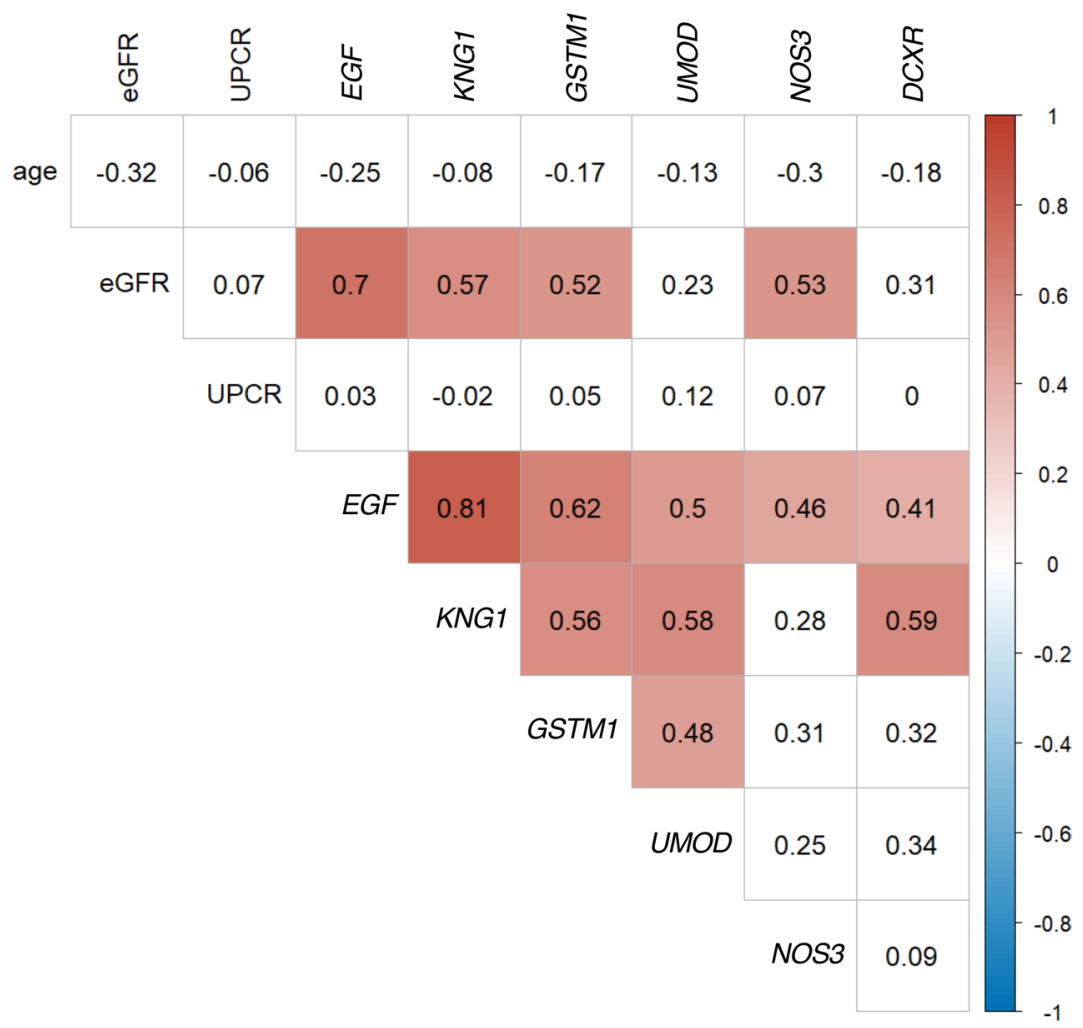

Figure 2. Correlations of continuous parameters in the discovery cohort $(n=63)$. Spearman's rank correlation coefficients are given for the 6 identified renoprotective factors and the clinical parameters age, eGFR, and UPCR. Significant positive and negative correlations after multiple testing corrections are highlighted in shades of red and blue, respectively. Spearman's rank correlation test was used to assess significance. Nonsignificant correlations are displayed with a white background.

gene expression data sets as well as data from the Human Protein Atlas. DCXR expression was found to be significantly higher (fold change $=3.36, P<0.001$ ) in the tubulointerstitium, as compared with glomeruli based on a published microarray data set in which renal tissue has been microdissected (7) (Figure 4A).

This is in line with data from the Human Protein Atlas indicating that $D C X R$ is abundant (on the protein level) in tubulointerstitium, whereas, almost no protein is found in the glomeruli (Figure 4B).

$D C X R$ also showed enriched mRNA expression patterns in human proximal tubular cells in comparison with other cell types based on data from a high-resolution single-cell RNA-seq data set, as shown in Figure 4C. DCXR expression was particularly elevated in S1, S2, and S3 tubular segments as shown in Figure $4 \mathrm{D}$ based on the second high-resolution RNA-seq data set under study.

Validation of $D C X R$ regulation in CKD in 3 independent transcriptomics data sets. $D C X R$ expression in tubulointerstitium was furthermore evaluated in 3 independent CKD gene expression data sets. A significant downregulation of $D C X R$ in diseased samples could be observed in all 3 cohorts under study. The largest $D C X R$ downregulation was observed in a data set of $5 \mathrm{CKD}$ samples, as compared with healthy control samples (fold change $=-12.91, P=0.004 ;$ Figure $5 \mathrm{~A}$ ). $D C X R$ was also significantly downregulated in tubulointerstitium in $10 \mathrm{DN}$ samples from the Woroniecka database (fold change $=-2.52, P=0.008$; Figure $5 \mathrm{~B}$ ) as well as in $10 \mathrm{DN}$ samples from the European Renal cDNA Bank (ERCB) (fold change $=-1.90, P=0.016$; Figure 5C).

Validation of DCXR's association with disease progression in the NEPTUNE cohort. The association of DCXR downregulation with the disease composite outcome was validated in patients with nephrotic syndrome from the large prospective multicenter and multiethnic NEPTUNE cohort. Basic characteristics of the NEPTUNE patients included in this study are provided in Table 2.

The tubulointerstitial expression of $D C X R$ showed a significant association $(P=0.0001)$ with disease outcome in Kaplan-Meier analysis (Figure 6A). Patients from the first tertile based on $D C X R$ values showed a significantly increased risk of progression compared with patients from the other 2 tertiles $(P=0.0023$ and $P=0.0002$, respectively, log-rank tests). 
A

DCXR expression across CKD diagnoses

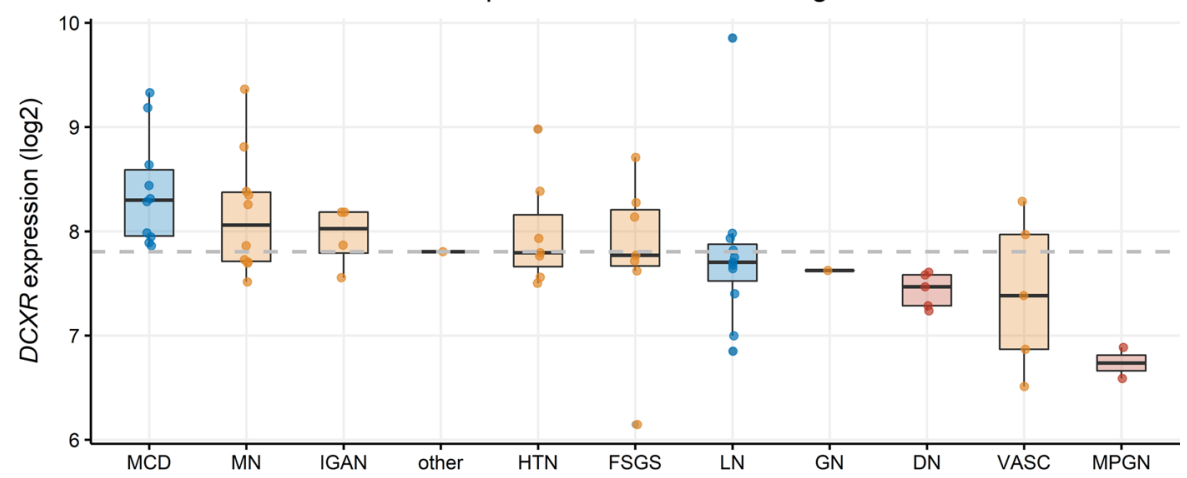

B

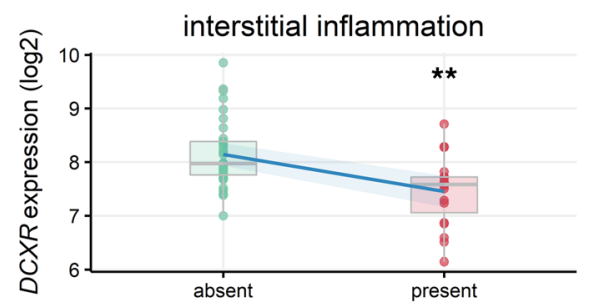

interstitial fibrosis
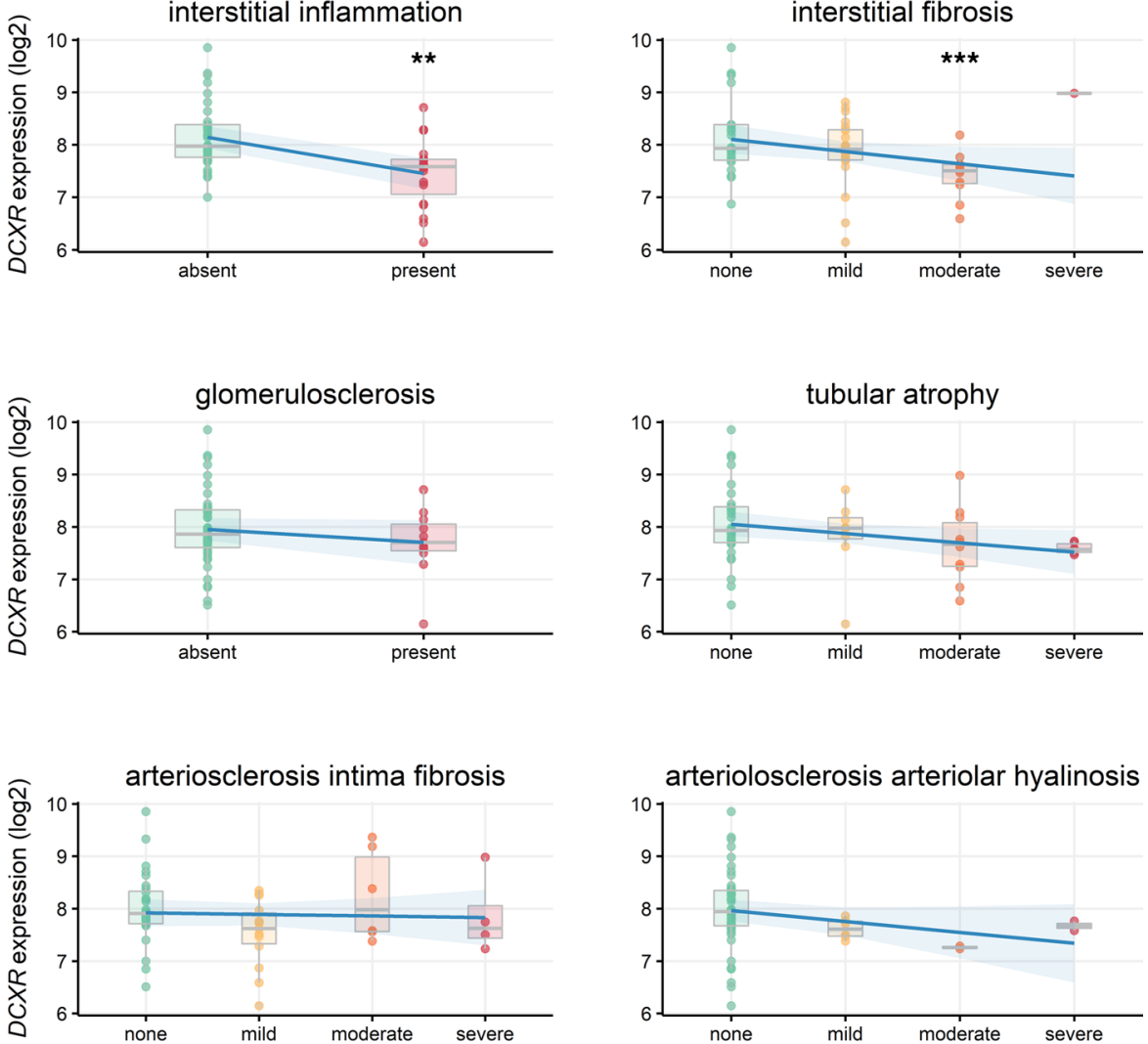

arteriolosclerosis arteriolar hyalinosis

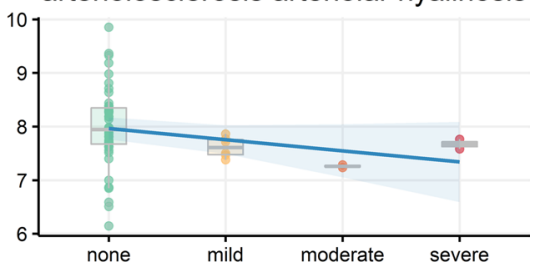

Figure 3. Association of DCXR with disease diagnosis and histological damage. (A) Gene expression levels for $D C X R$ are displayed across different CKD diagnoses. Median DCXR expression across all 63 samples is indicated by a dotted horizontal line. Diagnoses are sorted in descending order based on median DCXR expression levels. Diagnoses associated with poor outcome are highlighted in red (DN, MPGN), whereas diagnoses with favorable outcome are displayed in blue (MCD, LN). MCD, minimal change disease; MN, membranous nephropathy; MPGN, membranoproliferative glomerulonephritis; IGAN, IgA glomerulonephritis; HTN, hypertensive nephropathy; FSGS, focal segmental glomerulosclerosis; LN, lupus nephritis; GN, glomerulonephritis; DN, diabetic nephropathy; VASC, vasculitis. (B) DCXR expression levels across the different histological parameters. $t$ test, ${ }^{* *} P<0.05$; ANOVA, ${ }^{* *} P<0.05$.

Despite smaller sample and event size, lower $D C X R$ values were associated with worse outcome in the diagnosis-specific subgroup analysis in patients with focal-segmental glomerulosclerosis (FSGS) and MCD (Supplemental Figure 1).

$D C X R$ expression was positively correlated to baseline eGFR ( $r$ ho $=0.45, P<0.001$ ) and negatively correlated to UPCR (rho $=-0.27, P<0.001$ ) in NEPTUNE (Figure 6, B and C). DCXR expression was also associated with the degree of histological damage, as indicated by the significant negative 
A

$D C X R$

compartment specific expression

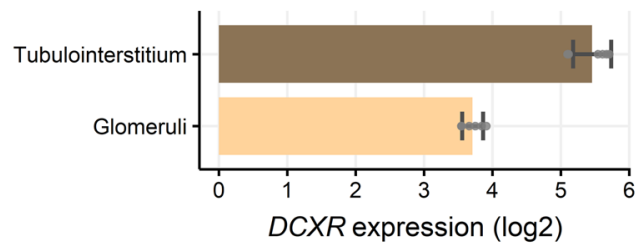

C

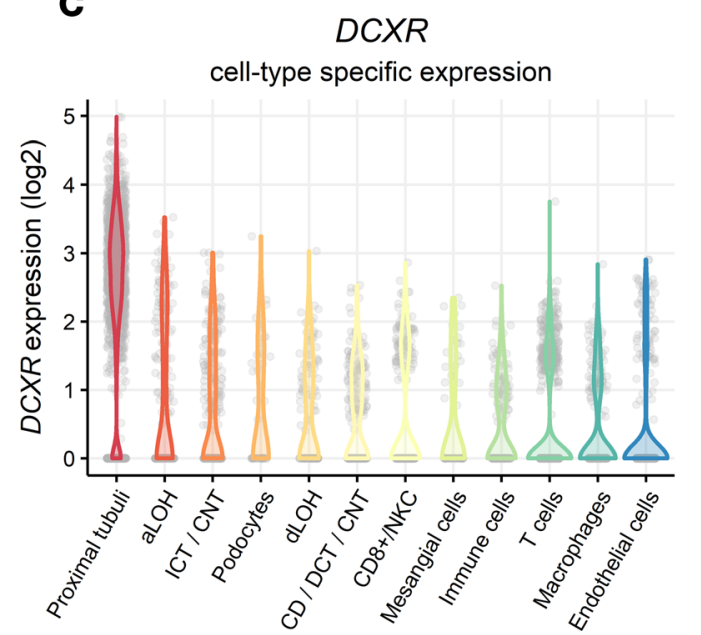

B

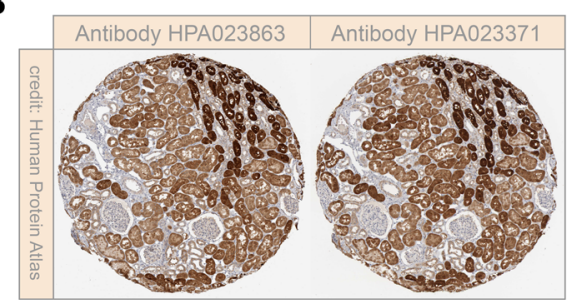

D

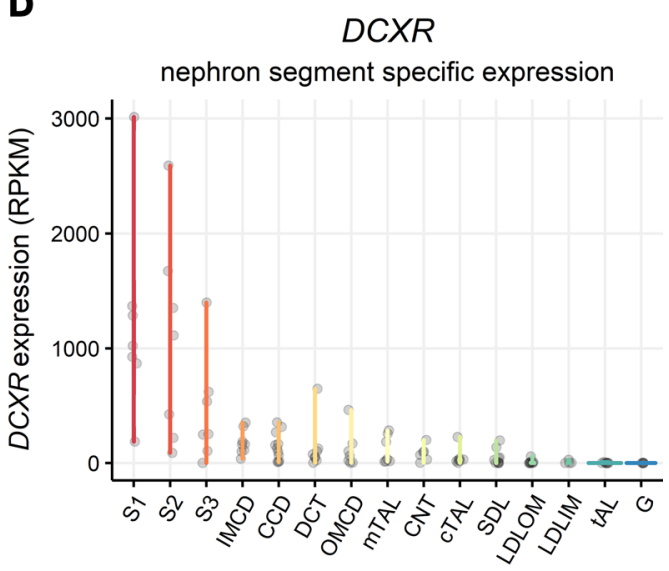

Figure 4. DCXR expression in renal tissue. (A) DCXR mRNA expression is significantly higher (fold change $=3.36, P<0.001$ ) in tubulointerstitium $(n=6)$ as compared with glomeruli $(n=6)$ based on $t$ test statistics. (B) DCXR protein abundance is also restricted to renal tubular cells. (C) Violin plots indicating enriched $D C X R$ expression in proximal tubule cells based on single-cell RNA-Seq experiments from human tissues. aLOH, ascending loop of Henle cells; ICT/CNT, intercalated/connecting cells; dLOH, distal/descending loop of Henle cells; CD/DCT/CNT, collecting duct/distal/ connecting cells; $\mathrm{CD}^{+} / \mathrm{NKC}, \mathrm{CD} 8^{+} /$natural killer cells. (D) Violin plots indicating that DCXR expression is mainly restricted to S1, S2, and S3 tubular segments. S1, S1 proximal tubule; S2, S2 proximal tubule; S3, S3 proximal tubule; IMCD, inner medullar collecting duct; CCD, cortical collecting duct; DCT, distal convoluted tubule; OMCD, outer medullary collecting duct; mTAL, medullary thick ascending limb; CNT, connecting tubule; cTAL, cortical thick ascending limb; SDL, short descending limb; LDLOM, long descending limb, outer medulla; LDLIM, long descending limb, inner medulla; tAL, thin ascending limb; G, glomeruli; RPKM, reads per kilobase million.

associations with the degree of interstitial fibrosis (rho $=-0.30, P<0.001$; Figure $6 \mathrm{D}$ ) and tubular atrophy (rho $=-0.30, P<0.001 ;$ Figure $6 \mathrm{E}$ ) respectively.

$D C X R$ as well as eGFR were associated with a worse prognosis in Cox regression analysis with hazard ratios of 0.44 and 0.49 , respectively. Age, tubular atrophy, interstitial fibrosis, and UPCR were also significant in Cox regression analysis (Figure 7).

Association of DCXR with members of the formation of xylulose-5-phosphate pathway and the dicarbonyl stress detoxification cascade. DCXR is member of the formation of xylulose-5-phosphate pathway. Tubular $D C X R$ expression was significantly positively associated with almost all members of this metabolic pathway, namely aldo-keto reductase family 1 member A1 (AKR1A1), crystallin lambda 1 (CRYL1), sorbitol dehydrogenase $(S O R D)$, as well as xylulokinase (XYLB; Figure 8).

We furthermore determined correlations of $D C X R$ expression with key enzymes involved in dicarbonyl stress detoxification. While renal $D C X R$ expression showed the strongest positive correlation to AKR1A1, the next strongest correlation was observed with hydroxyacylglutathione hydrolase $(H A G H)$, also known as glyoxalase 2. Interestingly, $D C X R$ showed no correlation to glyoxalase 1 (GLO1) and a negative correlation to aldo-keto reductase family 1 member $\mathrm{B}(A K R 1 B 1)$.

Effect of SGLT2 inhibition on tubular DCXR expression in vitro. We investigated the effect of the sodium glucose cotransporter-2 (SGLT2) inhibitor canagliflozin on $D C X R$ expression in human renal proximal tubular cells (HK2) that have been stimulated with high glucose. Canagliflozin significantly enhanced expression of $D C X R$ in HK2 cells by 1.28 -fold $(P=0.0194$; Figure $9 \mathrm{~A})$. The upregulation of $D C X R$ expression by SGLT2 inhibition was substantiated when extracting $D C X R$ expression levels from a recently published transcriptomics data set (8). The authors of this study have determined gene 
A

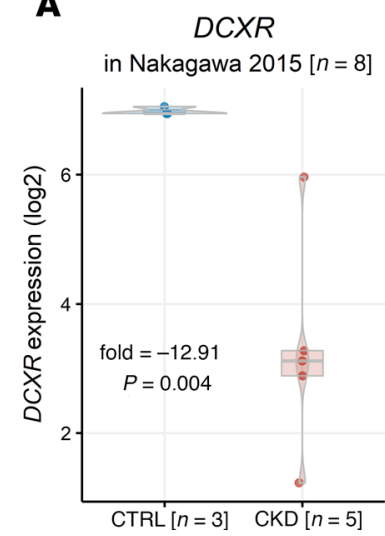

B

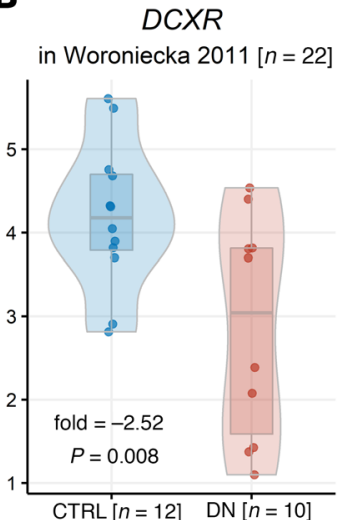

C

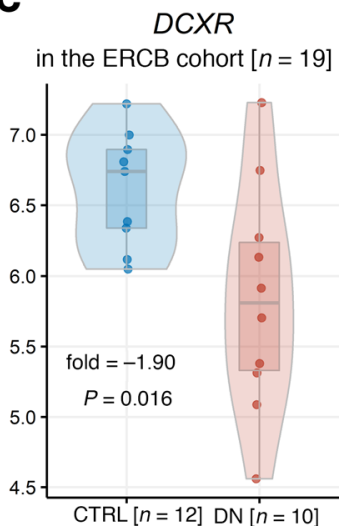

Figure 5. Validation of DCXR regulation in CKD in independent cohorts. $D C X R$ expression was found to be significantly downregulated in an independent CKD cohort (A) and 2 DN cohorts (B and C), as compared with healthy control subjects, when reanalyzing independent transcriptomics data sets. $P$ values are based on $t$ tests. CTRL, control group; CKD, chronic kidney disease; DN, diabetic nephropathy; ERCB, European Renal cDNA Bank.

expression profiles in 2 renal proximal tubular cells (RPTECs) studying the effect of the 2 SGLT2 inhibitors canagliflozin and empagliflozin. DCXR expression levels increased 1.70- and 1.84-fold in RPTECs as well as 2.35- and 2.22-fold in HK2 cells after canagliflozin and empagliflozin treatment, respectively (Figure 9B).

\section{Discussion}

This is, to our knowledge, the first study reporting on the association with disease progression of the renoprotective factor $D C X R$ in the context of human CKD. We show that (a) $D C X R$ expression in renal tissue is downregulated in CKD as compared with healthy controls; (b) DCXR expression is mainly restricted to S1, S2, and S3 renal tubular segments and also protein abundance is restricted to the tubular compartments; (c) $D C X R$ is associated with CKD outcome, a finding that was validated in studying the NEPTUNE cohort; (iv) $D C X R$ is associated with enzymes linked to dicarbonyl stress detoxification in human renal tubular tissue; and (d) $D C X R$ expression is upregulated in renal proximal tubular cells by SGLT2 inhibitors in vitro.

A number of clinical parameters has been reported to be associated with the course of disease development in CKD patients, including impaired renal function itself, hypertension, as well as the degree of proteinuria (9-11). Other risk factors associated with a worse prognosis are presence of cardiovascular disease or diabetes, black race, or being male (12).

Our discovery cohort of $63 \mathrm{CKD}$ patients seems to be representative, with significant associations to disease outcome observed for sex, baseline eGFR, and type of diagnosis. We decided to investigate this rather heterogeneous CKD patient population with different $\mathrm{CKD}$ diagnoses represented to increase our chances of detecting common renoprotective mechanisms and factors of general utility.

In addition to clinical parameters, a number of molecular biomarkers have been discovered, with the majority being elevated in the diseased state (13-16).

In this work, we were particularly interested in molecules with renoprotective properties instead of damaging potential. Of a previously published set of proteins with renoprotective potential (6), 6 are identified as predictors of CKD outcome in our discovery cohort. Evidence in the literature on the association with disease progression was available for EGF, KNG1, GSTM1, NOS3, and UMOD (17-26). The prognostic value of EGF has also been shown in the NEPTUNE cohort (18). GSTM1 and NOS3 transcript levels on the Affymetrix platform were too low in NEPTUNE samples for survival analysis. $K N G 1$ was significantly associated with disease progression in NEPTUNE ( $P=0.047$, log-rank test), whereas $U M O D$ was not significantly associated with outcome $(P=0.77$, log-rank test).

The focus of all further analyses and validation in the current study were subsequently on the sixth renoprotective factor, namely $D C X R$, for which no human data in the context of CKD disease progression is available. $D C X R$ is a member of the formation of xylulose-5-phosphate pathway with the main function of converting L-xylulose to xylitol. Deficiency in $D C X R$ is linked to pentosuria, a condition characterized by high L-xylulose levels in urine (27). We found strong positive correlations of tubular $D C X R$ expression 
Table 2. Patient characteristics of the NEPTUNE validation cohort

\begin{tabular}{|c|c|c|c|c|c|}
\hline & Cohort $(n=225)$ & FSGS $(n=68)$ & $\operatorname{MCD}(n=50)$ & MN $(n=45)$ & Other diseases $(n=62)$ \\
\hline Age (yr) & $34.9 \pm 21.9$ & $34.3 \pm 21.8$ & $21.5 \pm 20.9$ & $49.9 \pm 14.6$ & $35.4 \pm 20.5$ \\
\hline eGFR (ml/min/1.73 $\left.\mathrm{m}^{2}\right)$ & $84.5 \pm 43.1$ & $79.5 \pm 44.1$ & $112.3 \pm 44.0$ & $82.6 \pm 30.4$ & $70.1 \pm 40.8$ \\
\hline Follow-up time (years) & $2.4 \pm 1.7$ & $2.7 \pm 1.8$ & $2.5 \pm 1.8$ & $2.4 \pm 1.9$ & $1.9 \pm 1.4$ \\
\hline Female sex $[n(\%)]$ & $79(35)$ & $23(34)$ & $18(36)$ & $18(40)$ & $20(32)$ \\
\hline$D C X R$ (log2 expression) & $10.09 \pm 0.57$ & $10.06 \pm 0.51$ & $10.33 \pm 0.40$ & $10.15 \pm 0.47$ & $9.90 \pm 0.72$ \\
\hline RAAS blockade & $119(53)$ & $42(62)$ & $12(24)$ & $30(67)$ & $35(56)$ \\
\hline Antidiabetics & $8(4)$ & $4(6)$ & $0(0)$ & $2(4)$ & $2(3)$ \\
\hline Statins & $64(28)$ & $17(25)$ & $6(12)$ & $25(56)$ & $16(26)$ \\
\hline Steroids & $79(35)$ & $23(34)$ & $25(50)$ & $9(20)$ & $22(35)$ \\
\hline NSAIDs & $4(2)$ & $2(3)$ & $0(0)$ & $2(4)$ & $0(0)$ \\
\hline
\end{tabular}

Key clinical parameters of the NEPTUNE validation cohort are given for the whole cohort as well as for the different diagnosis subgroups. Mean values plus standard deviations are provided for continuous parameters. eGFR, estimated glomerular filtration rate; UPCR, urine protein to creatinine ratio; FSCS, focal segmental glomerulosclerosis; MCD, minimal change disease; MN, membranous nephropathy; RAAS, renin angiotensin aldosterone system; NSAIDs, nonsteroidal antiinflammatory drugs.

to other members of the formation of xylulose-5-phosphate pathway in our study. In the context of diabetes and kidney disease, another relevant renoprotective function is the ability to remove renal $\alpha$-dicarbonyl compounds under oxidative circumstances, among them glyoxal, methylglyoxal (MG), as well as 3-deoxyglucosone $(28,29)$. An abnormal accumulation of $\alpha$-dicarbonyls, known as dicarbonyl cell stress, can lead to cell and tissue dysfunction, as dicarbonyls are considered precursors of advanced glycation end products (AGEs; ref. 29-32). DCXR expression levels were significantly positively correlated with 2 key enzymes in the dicarbonyl stress detoxification cascade, namely $H A G H$, also known as GLO2, and AKR1A1. Based on these data and consolidated literature information, we speculate that $D C X R$ might offer a complementary way of counterbalancing dicarbonyl stress in human renal tubular cells (Figure 10).

The first clinical trials (NCT02095873) testing GLO1 inducers in the context of diabetes and associated vascular complications have already shown promising results $(33,34)$. Induction of $D C X R$ might, therefore, provide an alternative way of counterbalancing dicarbonyl stress to reduce generation of AGEs with a final beneficial effect on kidney function.

We were able to confirm the finding of reduced $D C X R$ mRNA expression levels in tubulointerstitium in CKD in 3 independent studies (Figure 5). We focused on $D C X R$ expression in tubulointerstitium, as $D C X R$ expression was significantly higher in tubulointerstitium as compared with glomeruli (Figure 4). Based on single-cell RNA-seq data, $D C X R$ expression is restricted to S1, S2, and S3 tubular segments (Figure 4C). The association of decreased $D C X R$ levels in renal tissue with disease outcome in the discovery cohort of 63 CKD patients was validated in an independent cohort consisting of 225 patients from the NEPTUNE study. The major driver of this significant association with disease outcome, in both the discovery and the validation cohorts, are patients in the first tertile of $D C X R$ expression levels, i.e., those patients with the lowest $D C X R$ levels (Figure 1 and Figure 6A). Patients with lower $D C X R$ levels have increased risk of progression to the composite endpoint in the NEPTUNE cohort (HR $=0.44$ [95\% CI: 0.29-0.67]). The association of lower DCXR levels with worse prognosis was observed in all disease subgroups of the NEPTUNE cohort (Supplemental Figure 1). The most significant association was found in the group of FSGS patients (the largest subgroup with 68 subjects). FSGS is one of the disease entities showing a large variance in the outcome parameter. The smaller sample sizes for the MN patient subgroup, as well as for the patient subgroup with other diseases, may have weakened the association with disease outcome. The association of $D C X R$ expression with clinical outcome and histological damage suggests that $D C X R$ is a marker mechanistically linked to renal damage and may, therefore, serve as a novel molecule for therapeutic intervention.

The renoprotective role of $D C X R$ was first postulated by Sudo and colleagues in an animal model where $D C X R$ was overexpressed (35). $D C X R$ was also found to be negatively associated with cortical 
A

$D C X R$

in the NEPTUNE cohort [ $n=225]$

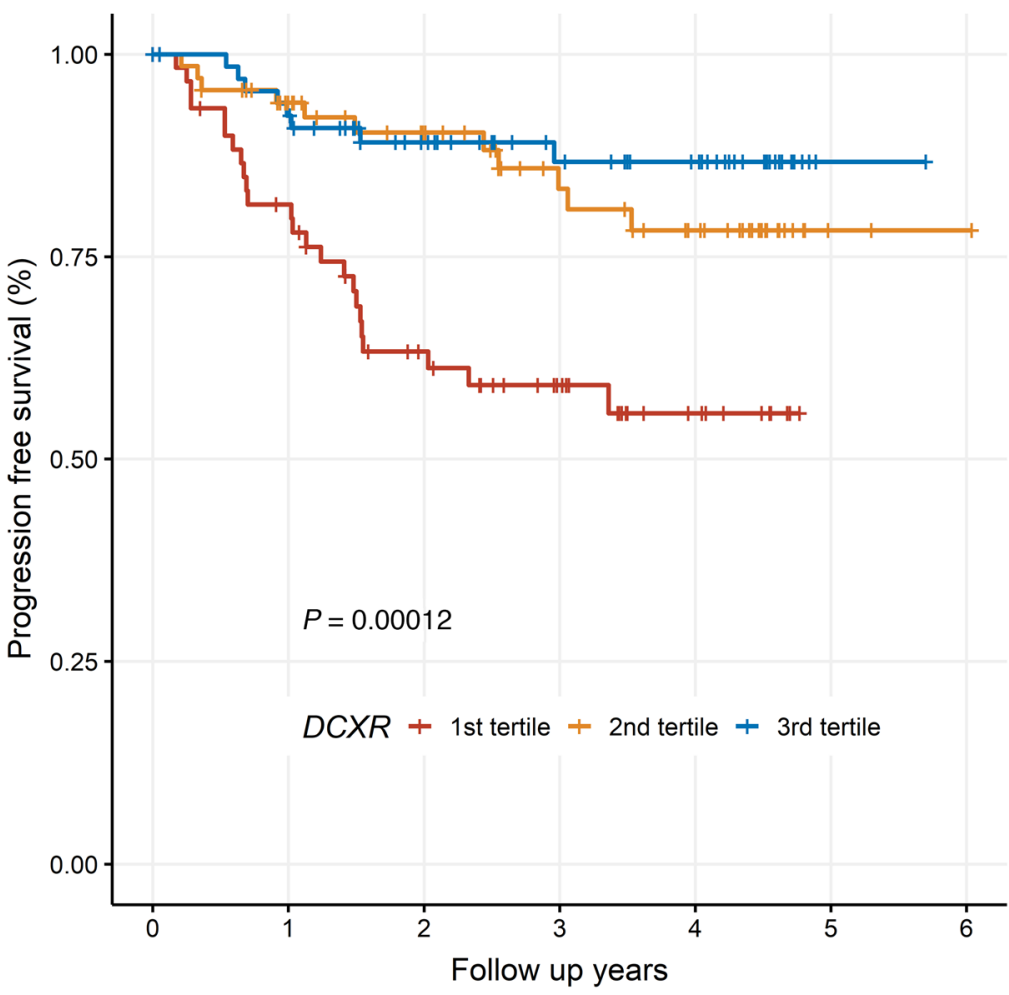

B

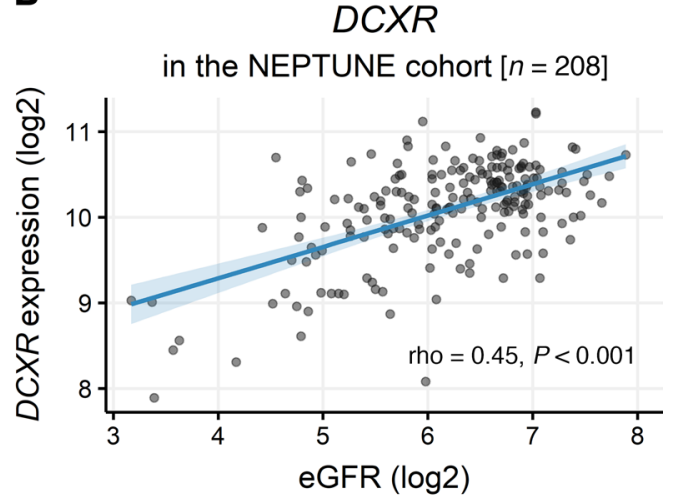

C $D C X R$

in the NEPTUNE cohort $[n=175]$

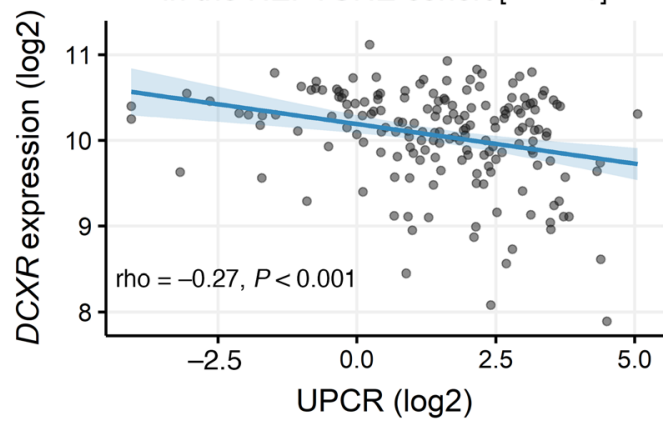

D

$D C X R$

in the NEPTUNE cohort $[n=203]$

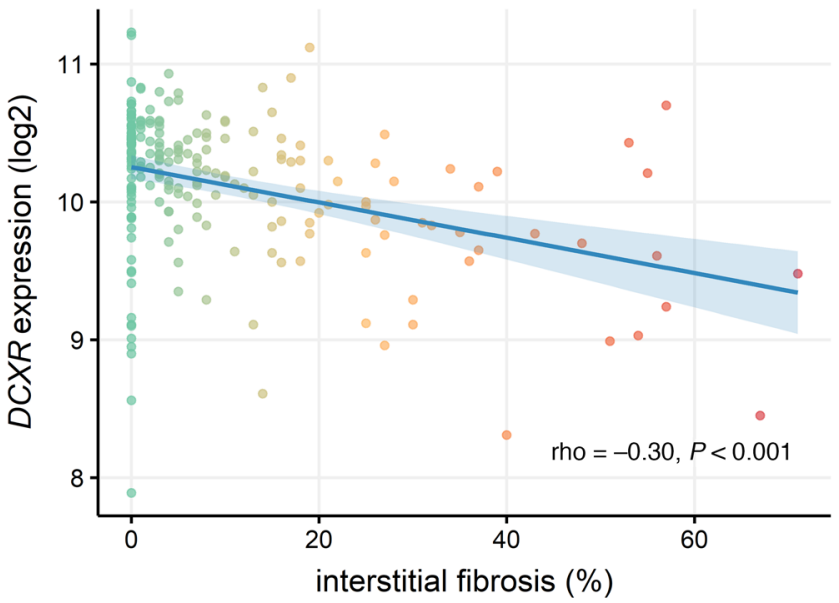

E

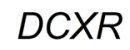

in the NEPTUNE cohort [ $n=203$ ]

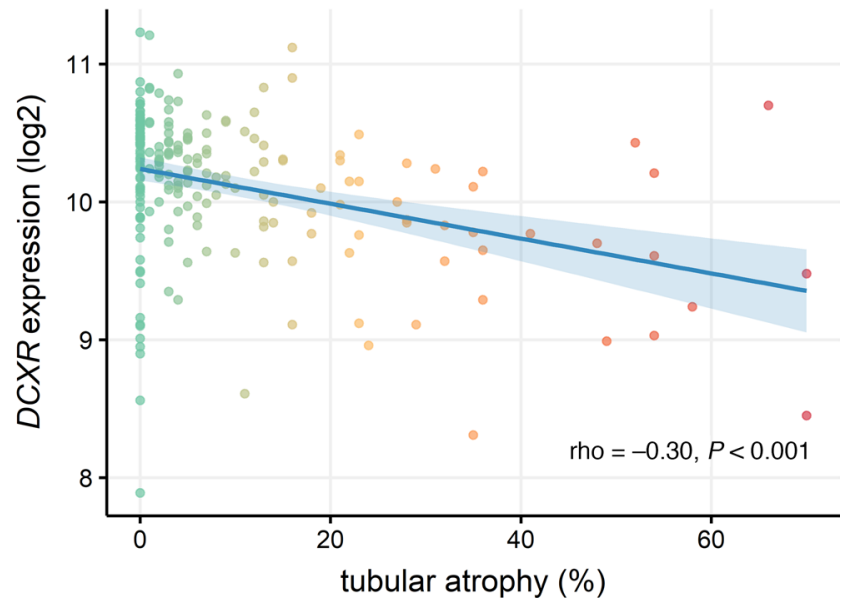

Figure 6. Validation of DCXR association with outcome in the NEPTUNE validation cohort. (A) DCXR expression in samples from the NEPTUNE validation study was significantly associated with outcome (B) as well as with baseline eGFR, (C) UPCR and the two histological parameters, (D and E) interstitial fibrosis and tubular atrophy. The $P$ value in the Kaplan-Meier analysis is based on the log-rank test statistics. $P$ values in plots B-E are based on Spearman's rank correlation test.

interstitial fractional volume by Nair and colleagues (36). In addition, calcineurin inhibitor treatment after renal transplantation had a detrimental effect on $D C X R$ gene expression based on a microarray study by Maluf and colleagues (37). DCXR protein concentration was also found to be upregulated around 2-fold in glomerular tissue from patients with diabetes mellitus who were being protected from development of diabetic kidney disease (38). Very recently, $D C X R$ was found in a proteomics study in a rat model to be 


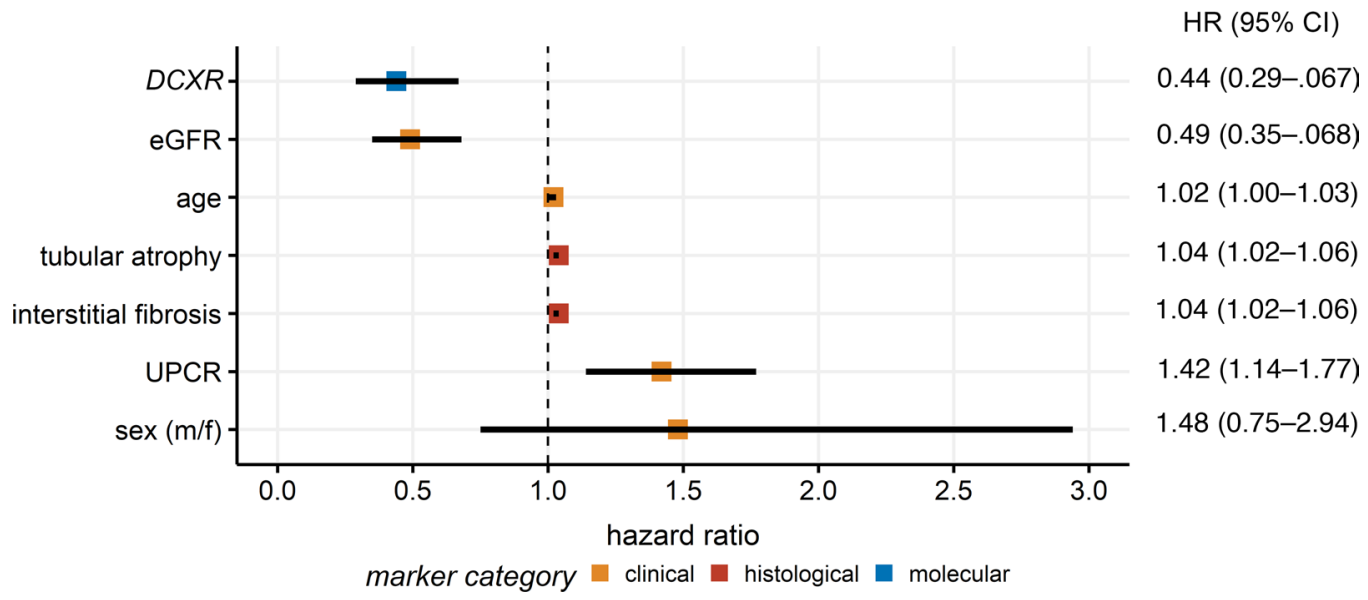

Figure 7. Hazard ratios for predicting disease progression in the NEPTUNE validation cohort. Hazard ratios and the $95 \%$ confidence intervals of molecular, clinical, and histological parameters are displayed for predicting the composite renal end point in the NEPTUNE validation cohort $(n=225)$ in univariate Cox regression analysis. All tested parameters except sex were significantly associated with disease outcome. The hazard ratio for $D C X R$ was 0.44 compared with a hazard ratio of 0.49 for eGFR. DCXR, dicarbonyl and L-xylulose reductase (log2 gene expression); eGFR, estimated glomerular filtration rate $\left[\log 2\left(\mathrm{ml} / \mathrm{min} / 1.73 \mathrm{~m}^{2}\right)\right]$; age in years; tubular atrophy and interstitial fibrosis in percentage of damaged tissue; UPCR, urine protein to creatinine ratio $[\log 2(\mathrm{~g} / \mathrm{g})]$.

downregulated in rat myocardium during development of type 2 diabetes (39). Overall, the evidence for $D C X R$ being involved in CKD progression is high, based on data from our study, as well as data from the scientific literature on the mechanistic involvement of $D C X R$.

We, furthermore, determined the effect of the SGLT2 inhibitor canagliflozin on DCXR expression in human renal proximal tubular cells in vitro. SGLT2 inhibitors are a class of antihyperglycemic drugs targeting the SGLT2 transporter in the S1 segment of the proximal tubule. SGLT2 inhibitors delay kidney function decline and have beneficial effect on cardiovascular outcome, although the exact underlying mechanisms are not fully understood (40-42). Canagliflozin, in our study, significantly upregulated DCXR expression levels by 1.28 -fold in HK2 cells that have been stimulated with high glucose. In a recently published transcriptomics data set the effect of the two studied SGLT2 inhibitors canagliflozin and empagliflozin was even higher, resulting in $D C X R$ expression increases of up to 2.35fold (8). Upregulation of $D C X R$ could therefore contribute to the renoprotective potential of SGLT2 inhibitors. $D C X R$ itself or associated metabolites in the mechanistic context of $D C X R$ deregulation might hold the potential to serve as predictive markers for SGLT2 inhibitors, which, however, needs to be determined in subsequent studies. Future mechanistic studies may also shed light on the link between SGLT2 inhibitors and dicarbonyl stress. Thus far, a beneficial effect of the SGLT2 inhibitor ipragliflozin on CCL2, ICAM1, and VCAM1 levels counterbalancing the induction by methylglyoxal has been reported in human umbilical vein endothelial cells (43).

Despite these congruent findings across different cohorts, this study has its limitations. Some CKD entities are not well represented in the discovery cohort and an analysis by CKD diagnosis was only feasible in the NEPTUNE validation cohort. Due to this composition of the discovery cohort, we might have missed some promising renoprotective factors with prognostic potential in specific CKD entities. We also only focused on those renoprotective factors being significantly downregulated in progressive $\mathrm{CKD}$ in this study.

Logical next steps in future studies are (a) to determine the potential of $D C X R$ and mechanistically linked metabolites in body fluids as noninvasive prognostic biomarkers for CKD progression and (b) to identify endogenous upstream regulators and compounds capable of inducing DCXR expression. There is evidence that $D C X R$ is detectable on the protein level in urine samples based on a mass spectrometry study by Adachi and colleagues in which they characterized the human urinary proteome (44).

In summary, in this study, we showed that a downregulation of $D C X R$ in renal tissue is associated with a poor prognosis in CKD. Mechanistically, this may be due to reduced capabilities to remove renal $\alpha$-dicarbonyl compounds that are prominent precursors of AGEs. Enhancing $D C X R$ expression may offer a strategy to counterbalance CKD progression with SGLT2 inhibitors, thereby, having a beneficial effect on $D C X R$ expression levels in renal proximal tubular cells. 
D-glucuronate

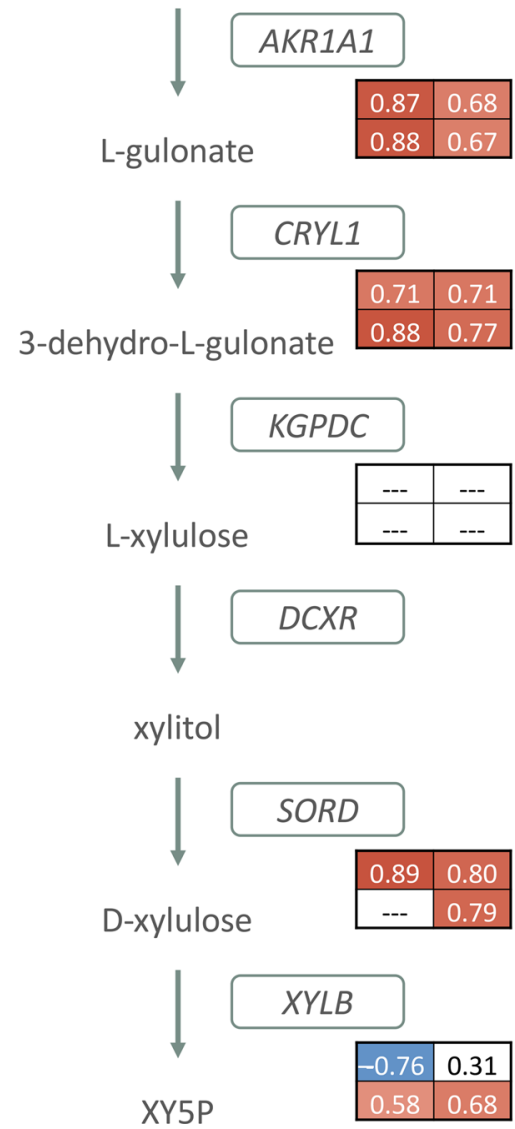

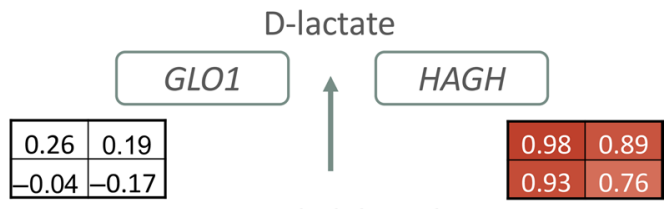

Methylglyoxal

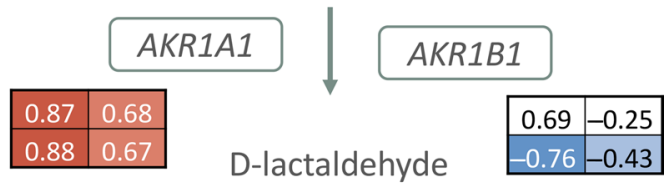

Legend:

Correlation to tubular DCXR expression in

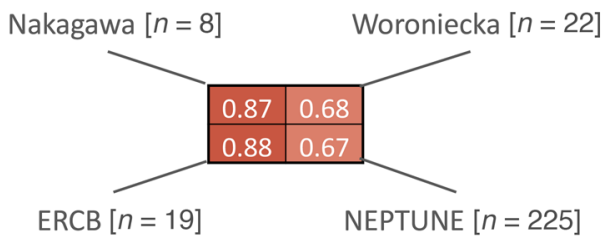

Pearson correlation

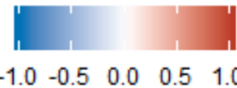

Figure 8. DCXR in the formation of xylulose-5-phosphate pathway and the link to dicarbonyl stress detoxification. Schematic representations of the formation of xylulose-5-phosphate pathway and key players in detoxification of dicarbonyl stress. Correlation coefficients to DCXR based on mRNA gene expression in the 4 renal tubular transcriptomics data sets used in this study are provided for key pathway members. Significant positive and negative correlations are highlighted in red and blue, respectively. Test statistic is based on Pearson's product moment correlation coefficient. Nonsignificant correlations are displayed with a white background.

\section{Methods}

Generating the set of renoprotective factors. In this study, we focused on a set of proteins previously reported to have renoprotective potential (6). This set has been consolidated and manually curated based on information in the literature via mining NCBI's Gene Reference into Function (GeneRIF) gene descriptions in the context of renoprotection and renal repair. BIRC5 (45), EGF (46), FGF1 (47), and CCN3 (48) were added to this set and also considered in the current analysis. Associations with renoprotection of these 4 proteins were not based on GeneRIFs but evidence from the literature (45-48).

The discovery CKD patient cohort. We determined the association with disease progression of renoprotective factor gene expression by reanalyzing a retrospective cohort of 63 patients with various $C K D$ diagnoses. Follow-up data from this patient cohort were updated in Q2 2018. Patients with a minimum follow-up time of 6 months who reached ESRD or experienced doubling of serum creatinine during the follow-up period were defined as progressive. Patients who did not develop ESRD or doubling of serum creatinine during follow-up with a minimum available follow-up time of 12 months were defined as stable. Clinical data from the last available follow-up visit were recorded in stable patients. For patients from the progressive group who started dialysis treatment, the last UPCR and serum creatinine values before initiation of dialysis were recorded. The Modification of Diet in Renal Disease Study (MDRD) formula was used to calculate eGFR values. Information on the degree of histological damage recorded by a pathologist was available for 55 patients. 
A

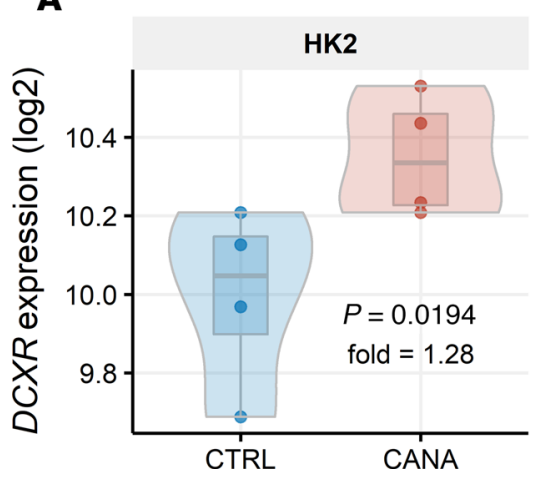

B

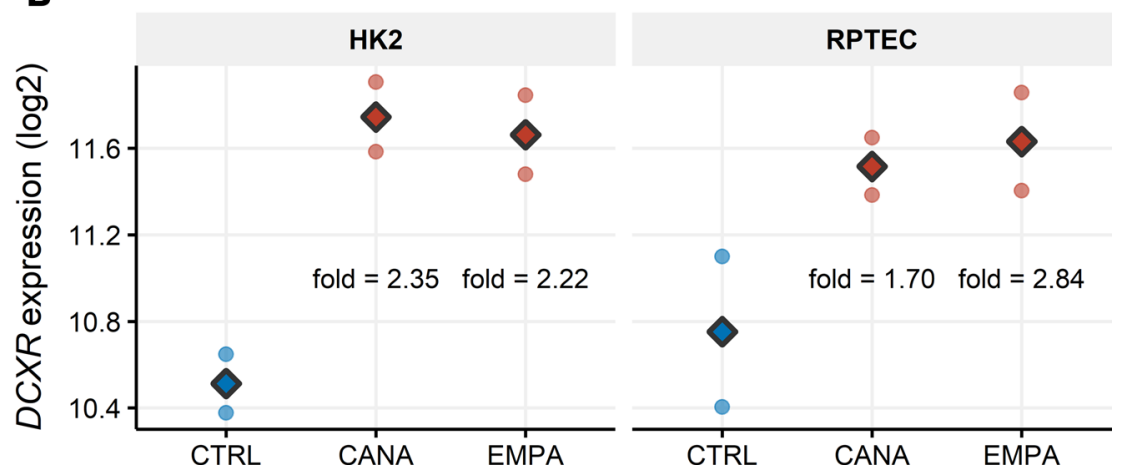

Figure 9. Effect of SGLT2 inhibitors on DCXR expression in renal proximal tubular cells. (A) Canagliflozin significantly upregulated $D C X R$ expression in high glucose stimulated renal proximal tubular HK2 cells $(n=4)$ as compared with untreated control samples $(n=4, P=0.0194, t$ test). (B) $D C X R$ expression was also found to be upregulated by both canagliflozin and empagliflozin in a published transcriptomics data set in RPTEC and HK2 cells with fold increases ranging from 1.70 to 2.35 (8). Diamonds indicate group mean expression values. CTRL, untreated control cells; CANA, canagliflozin; EMPA, empagliflozin.

Gene expression analysis of renoprotective factors in the discovery cohort. Gene expression data of the 63 discovery cohort patients, which have been deposited in NCBI's Gene Expression Omnibus (GEO GSE60861; ref. 5), were analyzed using the limma package of the statistical software framework R $(49,50)$. Preprocessing consisted of background correction, quantile normalization and summarization of probes with the same sequence spotted multiple times on the array. The SAM method was used to identify differentially expressed transcripts between stable and progressive patients, with FDR set as $<5 \%$. As the focus was on downregulated renoprotective factors in disease, we used a fold-change cutoff of $>1.25$ when comparing stable and progressive patients.

DCXR expression analysis in renal compartments. DCXR mRNA expression and protein abundance levels in renal compartments were investigated using data from kidney donors with normal renal function from a published gene expression study by Lindenmeyer and colleagues (7), as well as data from the Human Protein Atlas (51).

DCXR mRNA expression in different renal cell types or nephron segments was determined in 2 publicly available high-resolution single cell RNA-seq data sets. The first study published by Lee and

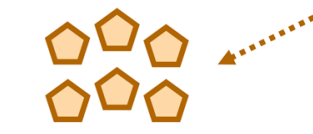

high urinary levels

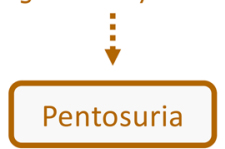

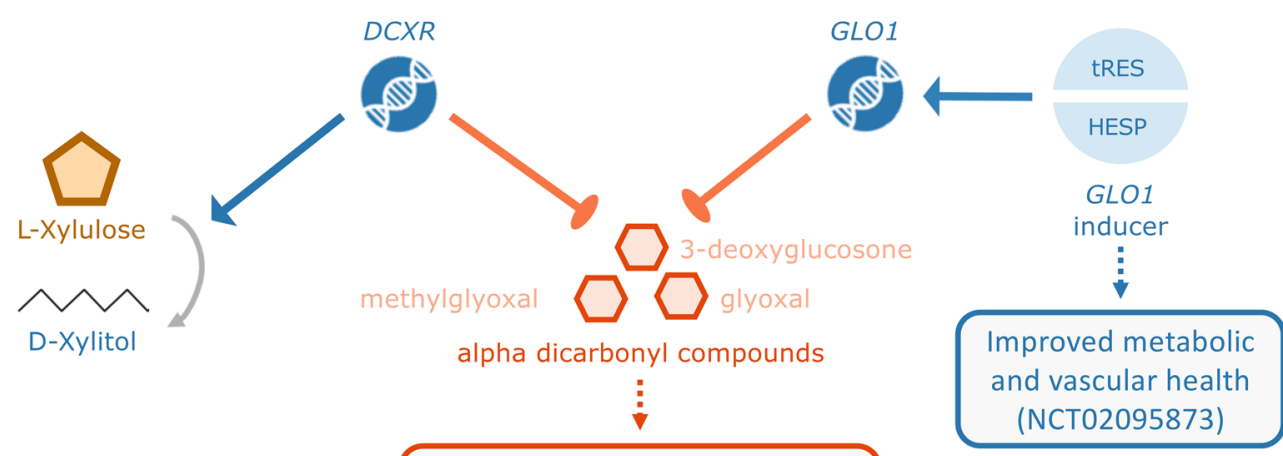

Precursors of AGEs

Inducers of fibrosis

Upregulated in uremic patients

Figure 10. Proposed model of DCXR's renoprotective role. Tubular DCXR converts L-xylulose to xylitol with high urinary L-xylulose levels being characteristic for the benign condition of pentosuria. DCXR was, furthermore, reported to degrade $\alpha$-dicarbonyl compounds such as glyoxal, methylglyoxal, or 3-deoxyglucosone. $\alpha$-Dicarbonyl compounds themselves are precursors of AGEs and thus detrimental to kidney function. GLO1 is another molecule degrading $\alpha$-dicarbonyl compounds and GLO1 inducers have been shown to positively effect metabolic and vascular health in obese patients in a phase I/II study (NCT02095873). t-RES, trans-resveratrol; HESP, hesperetin. 
colleagues provided comprehensive gene expression profiles in glomeruli and each of the 14 renal tubule segments after renal tubule microdissection from male Sprague-Dawley rats (52). The second expression study included 3 single-cell RNA-Seq data sets using adult tumor nephrectomy samples specifically harvested for single-cell analysis using 10X Genomics methodology (53).

Validation of reduced DCXR expression in patients with CKD. We extracted DCXR expression from 3 published independent transcriptomics data sets in the Nephroseq database (https://www.nephroseq.org/ resource/login.html). $D C X R$ expression in renal cortex and tubulointerstitial compartments was compared between CKD patients and healthy controls in data from Woroniecka et al. (54), Nakagawa et al. (55), as well as in samples from the ERCB cohort (18).

Validation of DCXR's association with disease progression. The association of DCXR expression with disease progression was assessed in samples with available gene expression profiles from the NEPTUNE cohort (56). NEPTUNE is a multicenter prospective cohort study of patients with proteinuric glomerular disease for which comprehensive clinical and molecular phenotyping data was collected at 21 sites (57). DCXR correlations to eGFR and UPCR, as well as to the 2 histological parameters, degree of interstitial fibrosis and degree of tubular atrophy, were determined using Spearman's rank correlation coefficient. The degree of interstitial fibrosis and tubular atrophy was assessed by at least 5 independent pathologists and given as the average percentage of obtained scores $(56,58)$. The association with disease progression was determined in time-to-event analysis using a composite endpoint of ESRD or 40\% reduction of baseline kidney function. Time-to-event analysis was also performed for individual CKD diagnosis groups. Univariate Cox regression analysis was subsequently used to determine hazard ratios for molecular, clinical, and histological parameters. Gene expression data used in this study are available within the Nephroseq database.

Correlation analysis to assess the mechanistic context of DCXR in human renal tubuli. DCXR is a member of the formation of xylulose-5-phosphate pathway (Reactome pathway R-HSA-5661270). The correlation of $D C X R$ mRNA expression to other pathway members was determined in the 4 human renal tubular gene expression data sets by Nakagawa et al. (55), Woroniecka et al. (54), ERCB (18), and NEPTUNE (56).

We furthermore investigated $D C X R$ correlations based on tubular mRNA expression patterns to key members of the dicarbonyl stress detoxification cascade.

In vitro gene expression analysis of SGLT2 inhibition on DCXR expression. Human proximal tubular kidney cells (HK2) were purchased from ATCC (CRL-2190) and cultured in Keratinocyte-Serum Free Medium (Thermo Fisher Scientific) containing 10\% fetal bovine serum, $5 \mathrm{ng} / \mathrm{ml}$ recombinant epidermal growth factor, $0.05 \mathrm{mg} / \mathrm{ml}$ bovine pituitary extract, $100 \mathrm{U} / \mathrm{ml}$ penicillin and $100 \mu \mathrm{g} / \mathrm{ml}$ streptomycin. Cells were grown at $37^{\circ} \mathrm{C}$ in a humidified atmosphere with $5 \% \mathrm{CO}^{2}$. Cells were serum deprived for 24 hours after grown to confluence. Cells were treated with $0.5 \mu \mathrm{M}$ canagliflozin or left untreated and subsequently stimulated with high glucose (30 mM). RNA was isolated after 24 hours with RNeasy Mini Kit (Qiagen) according to the manufacturer's protocol. RNA yield and quality were determined using a DS-11 FX+ spectrophotometer (DeNovix).

Gene expression profiling was done using Agilent Whole Human Genome Microarrays 4.44 v2 with 4 biological replicates in each study arm. Materials for microarray experiments were purchased from Agilent Technologies Inc. For each sample, 200 ng total RNA was used. Cyanine 3-labeled cRNA was generated with the Low Input QuickAmp Labeling Kit (5190-2305) for hybridization to oligonucleotide microarrays (Human GE 4x44K v2, G4845A). Arrays were scanned at $5 \mu \mathrm{m}$ using an Axon Gene Pix 4000B scanner (Molecular Devices) and signal intensity data were extracted using Agilent Feature Extraction software (v.9.5.3.1).

Preprocessing of transcriptomics data, including background correction, normalization, filtering, and summarization of identical sequence probes, was done using the limma R statistical package. Normalized expression levels of $D C X R$ were evaluated, comparing between untreated and canagliflozin treated samples. Microarray data have been deposited in NCBI's Gene Expression Omnibus database and are available with the accession number GSE106156.

We, furthermore, evaluated the effect of canagliflozin and empagliflozin on $D C X R$ expression in 2 human proximal tubular cell lines in a data set that became publicly available during the course of finalizing the present manuscript (8). In addition to gene expression in HK2 cells, we also investigated gene expression changes in RPTEs, a second widely used renal proximal tubular cell line. The lack of pretreatment with high glucose in prior studies was another difference in the experimental setup as compared with the cell culture experiments of the present study. 
Statistics. All analyses were done using R version 3.4.1. Spearman's rank correlation coefficient was used in correlation analysis between renoprotective factors and continuous clinical parameters. Bonferroni correction was used to adjust for multiple testing in the correlation analysis. A 2-tailed $t$ test was used for continuous, and $\chi^{2}$ test for discrete, clinical parameters when comparing the stable and progressive patient groups. ANOVA was used to identify significant associations of $D C X R$ with degree of histological damage. $P$ values of less than 0.05 were considered significant. The ggplot $2 \mathrm{R}$ package was used for generating box plots and scatterplots. The corrplot $\mathrm{R}$ package was used for creating the correlation plot.

The survival $\mathrm{R}$ package was used for Kaplan-Meier analysis using the log-rank test statistic. The survminer R package was used for generating Kaplan-Meier curves for continuous parameters using tertiles. For Cox regression analysis hazard ratios and 95\% confidence intervals were calculated.

Study approval. All biospecimens of the NEPTUNE cohort were procured after informed consent and with approval of the local ethics committees (see the Supplemental Acknowledgements for NEPTUNE enrolling centers). The University of Michigan Institutional Review Board approved gene expression studies (HUM0002468).

\section{Author contributions}

PP, GM, and MR designed and coordinated the study. MR and JK were responsible for clinical data collection of the discovery cohort. JL generated the gene expression data of the discovery cohort. PP performed the analyses in the discovery cohort with PP, WJ, RM, CZ, and MK performing data analysis of $D C X R$ in validation cohorts. PP drafted the first version of the manuscript with significant input from WJ and MR. All authors contributed to results interpretation and manuscript writing. All authors read and approved the final manuscript.

\section{Acknowledgments}

We thank all consortium partners of the BEAt-DKD (Biomarker Enterprise to Attack DKD; https://www. beat-dkd.eu) IMI2 project for fruitful discussions during project meetings. We also thank Heinz Regele and Andreas Kronbichler for their efforts in determining the degree of histological damage in samples from the discovery cohort. This project has received funding from the Innovative Medicines Initiative 2 Joint Undertaking under grant agreement no. 115974 (BEAt-DKD). This Joint Undertaking receives support from the European Union's Horizon 2020 research and innovation program and EFPIA and JDRF. This paper was also partially supported by NIDDK P30 DK081943 for the University of Michigan O'Brien Kidney Translational Core Center. The Nephrotic Syndrome Study Network Consortium (NEPTUNE), U54-DK-083912, is a part of the National Institutes of Health (NIH) Rare Disease Clinical Research Network (RDCRN), supported through a collaboration between the Office of Rare Diseases Research (ORDR), National Center for Advancing Translational Sciences (NCATS), and the National Institute of Diabetes Digestive and Kidney Diseases (NIDDK). Additional funding and/or programmatic support for this project have also been provided by the University of Michigan, the NephCure Kidney International, and the Halpin Foundation. See Supplemental Acknowledgments for NEPTUNE details.

Address correspondence to: Paul Perco, Department of Internal Medicine IV (Nephrology and Hypertension), Medical University Innsbruck, Anichstrasse 35, A-6020 Innsbruck. Phone: 43.0.512.504.81088; Email: paul.perco@i-med.ac.at.

1. Hill NR, Fatoba ST, Oke JL, et al. Global Prevalence of chronic kidney disease - a systematic review and meta-analysis. PLoS One. 2016;11(7):e0158765

2. Thomas B, et al. Global cardiovascular and renal outcomes of reduced GFR. J Am Soc Nephrol. 2017;28(7):2167-2179.

3. Liu Y. Cellular and molecular mechanisms of renal fibrosis. Nat Rev Nephrol. 2011;7(12):684-696.

4. Mayer G. Capillary rarefaction, hypoxia, VEGF and angiogenesis in chronic renal disease. Nephrol Dial Transplant. 2011;26(4):1132-1137.

5. Rudnicki M, Perco P, D'haene B, et al. Renal microRNA- and RNA-profiles in progressive chronic kidney disease. Eur J Clin Invest. 2016;46(3):213-226.

6. Perco P, Mayer G. Endogenous factors and mechanisms of renoprotection and renal repair. Eur J Clin Invest. 2018;48(5):e12914.

7. Lindenmeyer MT, et al. Systematic analysis of a novel human renal glomerulus-enriched gene expression dataset. PLoS One. 2010;5(7):e11545

8. Pirklbauer M, et al. Unraveling reno-protective effects of SGLT2 inhibition in human proximal tubular cells. Am J Physiol Renal Physiol. 2019;316(3):F449-F462. 
9. D'Amico G. Influence of clinical and histological features on actuarial renal survival in adult patients with idiopathic IgA nephropathy, membranous nephropathy, and membranoproliferative glomerulonephritis: survey of the recent literature. $A m J$ Kidney Dis. 1992;20(4):315-323.

10. Hladunewich MA, Troyanov S, Calafati J, Cattran DC, Metropolitan Toronto Glomerulonephritis Registry. The natural history of the non-nephrotic membranous nephropathy patient. Clin J Am Soc Nephrol. 2009;4(9):1417-1422.

11. Reich HN, Troyanov S, Scholey JW, Cattran DC, Toronto Glomerulonephritis Registry. Remission of proteinuria improves prognosis in IgA nephropathy. J Am Soc Nephrol. 2007;18(12):3177-3183.

12. Evans M, et al. Risk factors for prognosis in patients with severely decreased GFR. Kidney Int Rep. 2018;3(3):625-637.

13. Niewczas MA, et al. Circulating TNF receptors 1 and 2 predict ESRD in type 2 diabetes. J Am Soc Nephrol. 2012;23(3):507-515

14. Mayer G, et al. Systems biology-derived biomarkers to predict progression of renal function decline in type 2 diabetes. Diabetes Care. 2017;40(3):391-397.

15. Gerritsen KG, et al. Elevated urinary connective tissue growth factor in diabetic nephropathy is caused by local production and tubular dysfunction. J Diabetes Res. 2015;2015:539787.

16. Hayek SS, Quyyumi AA, Reiser J. Soluble urokinase receptor and chronic kidney disease. N Engl J Med. 2016;374(9):891.

17. Rudnicki M, et al. Hypoxia response and VEGF-A expression in human proximal tubular epithelial cells in stable and progressive renal disease. Lab Invest. 2009;89(3):337-346.

18. Ju W, et al. Tissue transcriptome-driven identification of epidermal growth factor as a chronic kidney disease biomarker. $S c i$ Transl Med. 2015;7(316):316ra193

19. Gonzalez-Calero L, et al. Urinary Kininogen-1 and Retinol binding protein-4 respond to acute kidney injury: predictors of patient prognosis? Sci Rep. 2016;6:19667.

20. Merchant ML, et al. Plasma kininogen and kininogen fragments are biomarkers of progressive renal decline in type 1 diabetes Kidney Int. 2013;83(6):1177-1184.

21. Tin A, et al. The Loss of GSTM1 associates with kidney failure and heart failure. J Am Soc Nephrol. 2017;28(11):3345-3352.

22. Nomani H, et al. Association between GSTM1, GSTT1, and GSTP1 variants and the risk of end stage renal disease. Ren Fail. 2016;38(9):1455-1461

23. Chang J, et al. Loss of GSTM1, a NRF2 target, is associated with accelerated progression of hypertensive kidney disease in the African American Study of Kidney Disease (AASK). Am J Physiol Renal Physiol. 2013;304(4):F348-F355.

24. Uyar M, et al. Endothelial nitric oxide synthase polymorphism influences renal allograft outcome. Clin Transplant. 2014;28(2):223-228.

25. Prajczer S, Heidenreich U, Pfaller W, Kotanko P, Lhotta K, Jennings P. Evidence for a role of uromodulin in chronic kidney disease progression. Nephrol Dial Transplant. 2010;25(6):1896-1903.

26. Bjornstad P, et al. Serum uromodulin predicts less coronary artery calcification and diabetic kidney disease over 12 years in adults with type 1 diabetes: the CACTI study. Diabetes Care. 2019;42(2):297-302.

27. Lee SK, Son le T, Choi HJ, Ahnn J. Dicarbonyl/1-xylulose reductase (DCXR): The multifunctional pentosuria enzyme. Int $J$ Biochem Cell Biol. 2013;45(11):2563-2567.

28. Odani $\mathrm{H}$, et al. Suppression of renal alpha-dicarbonyl compounds generated following ureteral obstruction by kidney-specific alpha-dicarbonyl/L-xylulose reductase. Ann N Y Acad Sci. 2008;1126:320-324.

29. Ebert B, Kisiela M, Maser E. Human DCXR — another 'moonlighting protein' involved in sugar metabolism, carbonyl detoxification, cell adhesion and male fertility? Biol Rev Camb Philos Soc. 2015;90(1):254-278.

30. Rabbani N, Thornalley PJ. Advanced glycation end products in the pathogenesis of chronic kidney disease. Kidney Int 2018;93(4):803-813

31. Rabbani N, Thornalley PJ. The critical role of methylglyoxal and glyoxalase 1 in diabetic nephropathy. Diabetes. 2014;63(1):50-52.

32. Giacco F, et al. Knockdown of glyoxalase 1 mimics diabetic nephropathy in nondiabetic mice. Diabetes. 2014;63(1):291-299.

33. Xue M, et al. Improved glycemic control and vascular function in overweight and obese subjects by glyoxalase 1 inducer formulation. Diabetes. 2016;65(8):2282-2294.

34. Rabbani N, Thornalley PJ. Glyoxalase 1 modulation in obesity and diabetes [published online ahead of print January 2, 2018]. Antioxid Redox Signal. doi: 10.1089/ars.2017.7424.

35. Sudo T, et al. Transgenic mice over-expressing dicarbonyl/L-xylulose reductase gene crossed with KK-Ay diabetic model mice: an animal model for the metabolism of renal carbonyl compounds. Exp Anim. 2005;54(5):385-394.

36. Nair V, et al. A molecular morphometric approach to diabetic kidney disease can link structure to function and outcome. Kidney Int. 2018;93(2):439-449.

37. Maluf DG, et al. Evaluation of molecular profiles in calcineurin inhibitor toxicity post-kidney transplant: input to chronic allograft dysfunction. Am J Transplant. 2014;14(5):1152-1163.

38. Qi W, et al. Pyruvate kinase M2 activation may protect against the progression of diabetic glomerular pathology and mitochondrial dysfunction. Nat Med. 2017;23(6):753-762.

39. Edhager AV, Povlsen JA, Løfgren B, Bøtker HE, Palmfeldt J. Proteomics of the rat myocardium during development of type 2 diabetes mellitus reveals progressive alterations in major metabolic pathways. J Proteome Res. 2018;17(7):2521-2532.

40. Wanner C, et al. Empagliflozin and progression of kidney disease in type 2 diabetes. N Engl J Med. 2016;375(4):323-334.

41. Neal B, et al. Canagliflozin and cardiovascular and renal events in type 2 diabetes. N Engl J Med. 2017;377(7):644-657.

42. Heerspink HJ, Perkins BA, Fitchett DH, Husain M, Cherney DZ. Sodium glucose cotransporter 2 inhibitors in the treatment of diabetes mellitus: cardiovascular and kidney effects, potential mechanisms, and clinical applications. Circulation 2016;134(10):752-772.

43. Salim HM, Fukuda D, Yagi S, Soeki T, Shimabukuro M, Sata M. Glycemic control with ipragliflozin, a novel selective SGLT2 inhibitor, ameliorated endothelial dysfunction in streptozotocin-induced diabetic mouse. Front Cardiovasc Med. $2016 ; 3: 43$.

44. Adachi J, Kumar C, Zhang Y, Olsen JV, Mann M. The human urinary proteome contains more than 1500 proteins, including a large proportion of membrane proteins. Genome Biol. 2006;7(9):R80

45. Chen J, Chen JK, Conway EM, Harris RC. Survivin mediates renal proximal tubule recovery from AKI. J Am Soc Nephrol. 2013;24(12):2023-2033. 
46. Yang CW, et al. Influence of the renin-angiotensin system on epidermal growth factor expression in normal and cyclosporine-treated rat kidney. Kidney Int. 2001;60(3):847-857.

47. Liang G, et al. Fibroblast growth factor 1 ameliorates diabetic nephropathy by an anti-inflammatory mechanism. Kidney Int 2018;93(1):95-109.

48. Riser BL, Najmabadi F, Garchow K, Barnes JL, Peterson DR, Sukowski EJ. Treatment with the matricellular protein CCN3 blocks and/or reverses fibrosis development in obesity with diabetic nephropathy. Am J Pathol. 2014;184(11):2908-2921.

49. Clough E, Barrett T. The Gene Expression Omnibus Database. Methods Mol Biol. 2016;1418:93-110.

50. Ritchie ME, et al. limma powers differential expression analyses for RNA-sequencing and microarray studies. Nucleic Acids Res. 2015;43(7):e47.

51. Uhlén M, et al. Proteomics. Tissue-based map of the human proteome. Science. 2015;347(6220):1260419.

52. Lee JW, Chou CL, Knepper MA. Deep sequencing in microdissected renal tubules identifies nephron segment-specific transcriptomes. J Am Soc Nephrol. 2015;26(11):2669-2677.

53. Gillies CE, et al. An eQTL landscape of kidney tissue in human nephrotic syndrome. Am J Hum Genet. 2018;103(2):232-244.

54. Woroniecka KI, Park AS, Mohtat D, Thomas DB, Pullman JM, Susztak K. Transcriptome analysis of human diabetic kidney disease. Diabetes. 2011;60(9):2354-2369.

55. Nakagawa S, et al. Molecular markers of tubulointerstitial fibrosis and tubular cell damage in patients with chronic kidney disease. PLoS One. 2015;10(8):e0136994.

56. Mariani LH, et al. Interstitial fibrosis scored on whole-slide digital imaging of kidney biopsies is a predictor of outcome in proteinuric glomerulopathies. Nephrol Dial Transplant. 2018;33(2):310-318.

57. Gadegbeku CA, et al. Design of the Nephrotic Syndrome Study Network (NEPTUNE) to evaluate primary glomerular nephropathy by a multidisciplinary approach. Kidney Int. 2013;83(4):749-756.

58. Barisoni L, et al. Digital pathology imaging as a novel platform for standardization and globalization of quantitative nephropathology. Clin Kidney J. 2017;10(2):176-187. 